\title{
Analysis and Management of Big Data Financial Risk
}

\author{
Lianyue He \\ Guizhou University of Finance and Economics, Guiyang 550025, China
}

\begin{abstract}
In recent years, people have improved the ability of data acquisition, storage, analysis and utilization, and big data comes into being.Big data include large capacity structured, semi structured and unstructured data that is difficult to store, analyze and manage in general database software or computer.Characteristics of big Data can be summarized as four Vs,volume,variety, velocity and value.Big data has great potential in the financial sector.Big data finance brings many conveniences to the financial industry, but at the same time, there are some risks and defects. This paper analyzes the risk of big data finance, and puts forward some strategies.
\end{abstract}

Big Data Financial; Risk; Strategy

\section{Keywords}

\section{大数据金融风险分析与管理}

\section{贺楝越}

贵州财经大学，贵阳 550025，中国

摘要：近年来, 人们对数据采集, 存储, 分析和利用的能力得到了显著提高, 大数据应运而 生。大数据是指一般数据库软件或者计算机难以储存、分析和管理的大容量结构化、半结构 化和非结构化数据, 有容量大、类型多、速度快、价值高等四个特点。大数据在金融方面有 巨大的运用潜力, 于是产生了大数据金融这一概念。大数据金融在为金融业带来许多便利的 同时，也存在着一些风险与缺陷。本文对大数据金融的风险进行了分析，并提出了几点策 略。

关键词：大数据金融；风险; 策略

\section{1. 大数据金融概述}

\section{1. 大数据定义}

随着科学水平的进步, 人们对数据 采集, 存储, 分析利用的能力得到了 显著提高。在数据数量大量增长的同
时，数据的内涵也由单纯的数字逐渐 扩大到影像、音频、日志、图片等范 围，也就是我们所说的 “大数据”。

“大数据” 这一概念, 最早由维克托・ 迈尔・舍恩伯格和肯尼斯・库克耶在编 写《大数据时代》中提出, 是指一般 
数据库软件或者计算机难以储存、分 析和管理的大容量结构化、半结构化 和非结构化数据。这些数据无法在有 限时间内用传统 IT 技术或软硬件感知 和处理, 这就对我们对其进行篮选利 用提出了更高层次的要求。

\section{2. 大数据特点}

大数据有以下四个特点: 容量 大、类型多、速度快、价值高。容量 大: 数据体量大, 数量级基本在 $\mathrm{TB}$ 到 $\mathrm{PB}$, 常规数据库软件很难实现对大数 据的管理，且数据规模会随着时间的 推移和技术的进步而增长。类型多: 数据类型多样, 来源也十分广泛, 包 括来自网络的日志、网页、图片, 来 自传感器的监测数据, 视屏、音频, 位置信息等。速度快：数据产生和更 新的速度快, 我们生活中接触的网 络、视屏监控等每分每秒都在产生巨 量的数据, 这也要求我们对大数据的 处理要有很高的时效性, 迅速给出处 理结果。价值高: 大数据的商业价值 高, 价值密度低。大数据体量巨大, 在繁杂的数据中, 有价值的信息十分 稀少, 需要经过挖掘、篮选和提炼, 才能得到对我们有用的信息。

\section{3. 大数据分析处理工具}

Facebook 的副总工程师曾经说 过: “如果你不利用所收集的数据, 那么你拥有的只是一堆数据, 而不是 大数据”。数据的收集在当今社会早 已不是什么难事, 但是大数据的关键 点并不在于数据的收集, 而在于通过 高效有用的方法对数据进行处理, 提 取茫茫数据海洋中对我们有用的信 息。在处理分析大数据时, 常用到的 工具有以下几种:

\section{(1) Hadoop}

Hadoop 是一个能够让用户轻松架 构和使用的分布式计算平台。用户可
以轻松地在 Hadoop 上开发和运行处理 海量数据的应用程序。它主要有高可 靠性、高拓展性、高效性和高容错性 等优点 ${ }^{[1]}$ 。

\section{(2) Storm}

Storm 是自由的开源软件, 是一个 分布式的、可扩展的、容错的实时计 算系统, 很容易设置和操作。Storm 可 以非常可靠的处理庞大的数据流, 可 用于处理 Hadoop 的批量数据。Storm 有许多应用领域：实时分析、在线机 器学习、不停顿的计算、分布式 RPC、 ETL 等等。Storm 的处理速度很快, 每 个节点每秒钟可以处理 100 万个数据 元组 ${ }^{[1]}$ 。

(3) RapidMiner

RapidMiner 是世界领先的数据挖 掘解决方案, 有很大程度上的先进技 术。它的数据挖掘任务涉及范围广 泛, 包括各种数据艺术, 简化数据挖 掘过程的设计和评价。它的数据挖掘 过程简单, 结果强大直观, 可提供多 层次的数据视图, 确保有效和透明的 数据, 同时可以用简单脚本语言自动 进行大规模进程。现在已成功的应用 在包括文本挖掘, 多媒体挖掘, 功能 设计, 数据流挖掘, 集成开发的方法 和分布式数据挖掘等许多不同的应用 领域 ${ }^{[1]}$ 。

对于其他的大数据分析处理工具 在此就不过多的加以赘述了。

\section{4. 大数据金融}

我们的生活离不开网络, 各项活 动也均在产生数据, 这些数据被各种 传感器感知、收集就形成了大数据。 我们关心的信息, 对于事物的偏好, 日常发生的活动, 这些都在日益不间 断产生的数据中有迹可循。并且这些 数据是无意识而全面的, 这就确保了 信息的完整性和真实性。企业若将这 
些宝贵的信息加以提炼运用，就可以 在很大程度上减少与客户之间的信息 不对等问题，达到 “投其所好” 的效 果。

大数据金融, 是指建立在大规模 数据信息上的金融行为, 即集合海量 非结构化数据, 通过大数据、互联 网、云计算等信息化处理方式，结合 传统金融服务，开展资金融通、创新 金融等服务。

大数据金融与传统金融相比, 有 着难以比拟的优势。在大数据的加持 下，企业能够更加贴近客户，了解客 户需求, 通过对不同的客户群体提供 差异化的服务来提高客户的价值, 实 现非标准化的个性化服务, 注重提高 客户体验, 增加客户粘性。

比如, 信贷企业可以通过对客户 消费数据进行实时分析, 以极低的成 本得到关于客户的全面信息。通过分 析和挖掘客户的相关交易信息, 掌握 客户的消费习惯和行为偏好, 了解客 户的信用等级以及资金状况, 以准确 地预测客户行为, 对潜在客户进行挖 掘与篎选, 提高服务的效率, 降低企 业在信息不对称情况下存在的信贷风 险。

在商品制造企业推行新产品时, 不再需要冒着巨大的风险, 通过很长 一段时间的市场反馈数据来判断商品 价值与接受度, 而可以结合搜索引擎 中商品的搜索频率, 网站页面下客户 的反馈评价等各种数据, 在短时间内 判断商品在市场中是否能获得客户的 认可, 是应该及时止损还是继续生产 相关商品。

目前, 大数据服务平台的运营模 式可以分为以阿里小贷为代表的平台 金融模式和京东、苏宁为代表的供应 链金融模式。平台金融模式主要工作 内容企业是对企业长期以来积累的大 数据通过互联网、云计算等信息化方 式对其数据进行专业化的挖掘和分
析, 提供平台实现需求方与供给方的 对接, 实现金融交易。供应链金融模 式, 是核心龙头企业依托自身的产业优 势地位, 通过其对上下游企业现金流、 进销存、合同订单等信息的掌控, 依托 自己资金平台或者合作金融机构对上 下游企业提供金融服务的模式。

\section{2. 大数据金融的风险}

\section{1. 信息泄露风险}

由于我们的生活与数据密切相 连, 在这个被无数传感器包围的环境 里, 我们的各种信息无时不刻在被记 录着, 这些信息在方便企业对我们的 消费习惯, 行为偏好进行了解和预测 的同时, 也可能造成信息的过度挖 掘, 消费者隐私的泄露, 以及信息被 不法分子肆意利用, 侵害消费者利益 等情况的产生。

消费者的信息可以大致分为两 类：基本信息和账户信息。基本信息 主要包括投资者的姓名、电话、邮 箱、家庭住址、身份证号, 社交软件 号等。这些信息虽然公开程度比较 高, 但是若被人恶意使用, 很可能导 致消费者被骚扰电话、短信、邮件困 扰, 同时存在着社交软件被人盗用, 用于欺诈消费者亲友, 骗取钱财的风 险。账户信息主要包括消费者在金融 机构开立的账户名称、密码、资产构 成和交易明细等。这些信息如果泄 露, 会威胁到消费者金融资产的安 全, 可能出现盗用账号, 擅自变卖金 融产品, 设置陷阱或直接盗取消费者 资金财产等恶果。

这些信息的泄露使得消费者的隐 私权得不到保障，不仅会让消费者损 失钱财, 更会使金融市场混乱动荡, 不利于金融业的健康发展。 


\section{2. 信息不对称风险}

前面说到大数据解决了需求方和 供给方信息不对称的风险，这是站在 正常合法交易角度上提出的。大数据 使消费者的需求有意或无意的被记录 在数据中, 使供给方能够更轻松全面 的挖掘需求方的各种需求, 从而更注 重消费者感受, 提供更个性化的服 务。这显然促进了金融行业的发展。

然而, 站在消费者的角度, 需求 方与供给方掌握的信息并不是对等 的。供给方是强势的一方，他们可以 利用更多的资源，采取多种手段，通 过专业的分析方法和工具, 通过大数 据对消费者进行全方位的分析, 了解 他们关注的信息, 洞察他们的行为偏 好, 甚至可以准确的预测消费者的行 为, 这使得消费者在他们面前近乎透 明。而消费者很少能拥有行业资源, 获得的数据与供给方相比不值一提, 并且消费者通常不具备进行大数据分 析的经济条件和专业技术，这使得消 费者在交易中处于弱势。

利用这些优势，不法供应商可以 在交易中，提供消费者感兴趣的虚假 投资信息，通过消费者的行为偏好诱 导消费者掉入陷阱。这就出现了平台 获取投资人大量资金后突然倒闭，消 费者投资落空的事件。

\section{3. 法律监管缺失风险}

我国大数据金融发展时间并不 长, 涉及的范围广泛, 目前现有的法 律法规和现有的监管体制都不能很好 的适用于大数据金融。与金融交易相 关平台的虽然基本要求实名制, 但是 由于管理制度尚未完善, 依然无法杜 绝计骗等非法行为。再者, 对于金融 平台是否需要取得合法营运证明, 如 何保证消费者资金安全等问题也没有 得到很好的解决。存在的诸多问题使 得大数据金融在交易双方出现利益受
损陷入纠纷时难以得到法律的支持与 保护。

\section{3. 大数据金融风险管理对策}

\section{1. 加强信息保密力度}

我们可以对消费者的关键核心数 据以及较大金额交易明细进行特殊加 强保护, 这样即使信息泄露, 也可以 避免泄露关键信息, 尽最大可能减少 信息泄露给消费者带来损失。通过这 一有针对性的管理手段, 能大大降低 用户消费数据泄露和资金损失风险。 另外我们应该加强立法, 健全我国金 融隐私信息保护制度。要通过立法, 大幅度提高泄露用户隐私的违法成 本，从根本上遏制盗卖个人信息的行 为, 逐步建立并完善有关用户隐私信 息保护的法律体系。最后, 我们应该 完善客户信息泄露的应急处理方案。 除了从预防的角度制定规章制度之 外，对于客户信息外泄的应急处理方 案也要有相应的明文规定, 以便明确 按照法律规定追究信息泄露者的责 任, 并按一定的标准给信息被泄露的 用户合理的赔偿。

\section{2. 加强相关知识普及}

虽然近年来大数据金融发展迅 速, 但是大多数普通人对互大数据金 融的了解并不多, 投资风险的防范意 识十分薄弱, 所以我们应该重视对大 数据金融知识的宣传, 提高人们对大 数据金融风险的认识。我们可以通过 广播、新闻、网络等平台, 对出现大 数据金融纠纷的案例进行报导, 邀请 专家进行分析讲解, 来提高消费者的 风险防范意识。银行等机构可以不定 期通过手机短信或电子邮件的方式为 客户发送金融平台安全使用知识及近 期常见金融诈骗手段等信息，来提醒 客户保持警惕, 养成正确的自我保护 
保护意识。另外，金融机构可以定期 印制专门的宣传册来加强用户信息保 护的宣传力度。

\section{3. 加强法律管理规范}

大数据金融的快速发展已经无法 用现有法律制度来进行监管了, 且大 数据金融涉及范围太广泛，应该由什 么机构来对其进行监管也成为当前一 大难题。当前由于法律、法规的不健 全, 许多大数据金融交易, 实质上已 经难以辨别究竟是合法还是非法。在 这样的状况下, 完善大数据金融的监 管和法律体系就显得十分迫在眉睫。 首先, 要进一步加强入行业资格的审 查与鉴定，防止出现非法经营的现 象, 减少出现金融诈欺的可能性; 其 次, 在现有法律法规的基础上, 要充 分结合大数据金融发展前景预测, 尽 可能使法律法规适用于更多的情况， 不至于在大数据金融发展过程中频繁 修改。最后, 进一步加大对大数据金 融消费者的权益保障力度, 因为消费 者在交易中处于弱势，相关法律法规 应适当向消费者的利益倾斜, 以维护 市场秩序，促进大数据金融健康有序 发展。

\section{4. 总结}

大数据时代的到来使得我们获取 的信息数量不再只是过去的部分抽 样, 而是能够以完整的全部数据为对 象, 通过一些科学的方法和专门的工 具, 篮选、提炼出我们需要的信息。 而这些信息是我们从前无法获得的， 且由于留下信息者的无意性, 这些信 息是真实而有价值的。大数据金融是 我国未来金融发展的必然方向, 这些 珍贵的信息为金融行业的发展提供了 十分有力的支持。

但是大数据为金融行业带来的不 止是便利, 也存在着信息容易泄露,
消费者与不法交易者信息不对称导致 利益损失以及缺乏法律保护等风险。 对此, 我们应该加强平台对消费者信 息保密的力度, 通过宣传提升消费者 的自我保护意识, 同时尽快完善相关 法律法规, 争取尽快为大数据金融的 发展创造一个稳定、健康的市场环 境, 促进我国经济蓬勃发展。

\section{参考文献}

[1] http://www.thebigdata.cn/JiShuBoKe/ 9164.html.

[2] 林巍、王祥兵，大数据金融：机 遇、挑战和策略, 金融经济, 2016 .

[3]刘晓曙，大数据时代下金融业的发 展方向与趋势及其应对策略, 科学 通报, 2015 . 